\title{
Genistein ameliorates parathyroid hormone-induced epithelial-to-mesenchymal transition and inhibits expression of connective tissue growth factor in human renal proximal tubular cells
}

Yunshan Guo', Aiping Zhang', Yaohai Ding1, Yanxia Wang', Weijie Yuan²

1Department of Nephrology, General Hospital of Jinan Military Jinan, Shangdong, China 2Department of Nephrology, Shanghai First People's Hospital Affiliated Shanghai Jiaotong University, Shanghai, China

Submitted: 24 February 2012

Accepted: 14 June 2012

Arch Med Sci 2013; 9, 4: 724-730

DOI: 10.5114/aoms.2013.36929

Copyright (c) 2013 Termedia \& Banach
Corresponding author:

Weijie Yuan

Department of Nephrology

Shanghai First People's

Hospital Affiliated

Shanghai Jiaotong University

201101 Shanghai, China

Phone: 021-63819323

E-mail: yuanweijie2011

@126.com

\begin{abstract}
Introduction: Genistein, a soybean and soy-based product, has been reported to inhibit the growth of a wide range of cancer cells, but there is no evidence concerning its treatment of chronic kidney disease. The aim was to investigate whether genistein has potential to inhibit parathyroid hormone (PTH)-induced renal interstitial fibrosis.

Material and methods: Using human renal tubular epithelial HK-2 cells, $\alpha$-smooth muscle actin ( $\alpha-S M A)$ was assessed by using immunofluorescence detection. $\alpha$-Smooth muscle actin, E-cadherin and connective tissue growth factor (CTGF) were measured by Western blot analysis. The promoter activity of the CTGF gene was examined by the luciferase reporter assay.

Results: When cells were treated with PTH $(0.1 \mathrm{nM})$ for $48 \mathrm{~h}, \alpha$-SMA protein expression was induced significantly, the protein expression of E-cadherin decreased substantially, and the promoter activity of the CTGF gene as well as its mRNA and protein expression levels increased $(p<0.01)$. Interestingly, genistein effectively inhibited PTH-induced $\alpha$-SMA expression, restored E-cadherin expression, decreased mRNA and protein expression of CTGF, and suppressed the promoter activity of CTGF in a dose-dependent manner.

Conclusions: Genistein has the ability to block the biomarker for renal transdifferentiation and epithelial-to-mesenchymal transition, $\alpha$-SMA, following PTH treatment and inhibit CTGF expression in human renal tubular epithelial cells; these might be important modes of actions that contribute to genistein antifibrogenic effects and may have great implications for its potential in clinical treatment of renal interstitial fibrosis.
\end{abstract}

Key words: genistein, parathyroid hormone, transdifferentiation, connective tissue growth factor, renal tubular epithelial cell.

\section{Introduction}

The progression of chronic kidney disease is characterized by continuous accumulation and deposition of extracellular matrix (ECM) resulting in widespread tissue fibrosis. It is generally believed that renal interstitial fibrosis (RIF) is the common characteristic of chronic kidney disease leading to end-stage renal failure, a devastating condition requiring life-long treatment with dialysis or renal transplantation [1]. 
$\alpha$-Smooth muscle actin ( $\alpha$-SMA) in positive myofibroblasts, a major source of forming fibroblast and main cells constituting ECM, has been associated with RIF and chronic renal disease [1]. Through transdifferentiation of $\alpha$-SMA positive myofibroblasts, renal tubular epithelial cells produce a series of inflammatory and fibrogenic cytokines such as transforming growth factor $\beta 1$ (TGF- $\beta 1$ ) and connective tissue growth factor (CTGF), which participate in further development of RIF [2]. Transforming growth factor- $\beta 1$ is a profibrotic regulator that can stimulate tubule epithelial cells to undergo epithelial mesenchymal transdifferentiation, while CTGF has been recognized as one of the important downstream factors that mediate the fibrotic activity of TGF- $\beta 1$ [3] and as a common factor involved in RIF [4]. Furthermore, studies have indicated that tubular epithelial cells undergo a critical transformation, epithelialto-mesenchymal transition (EMT) [5]. It has been shown that fibroblasts arise in large numbers (42\%) locally by EMT [6], and such transdifferentiating epithelial cells in renal tubules may play a fundamental role leading to eventual renal damage.

For clinical renal disease, the most significant advance in EMT studies may lie in the identification of a possible therapeutic intervention of cellular transdifferentiation and fibrogenesis to block renal fibrotic lesions. The isoflavone genistein (4,5,7-trihydroxy isoflavone) is a naturally occurring phytoestrogen found in soybeans. Extensive epidemiological and animal studies as well as in vitro experiments have been performed indicating that genistein exerts beneficial effects in a multitude of diseases, including cancer [7] and cardiovascular-related illness [8]. In addition, genistein has been shown to down-regulate cytokine-induced signal transduction events in the cells of the immune system [9]. However, at present, there is no direct study addressing whether genistein can exhibit an effect on the inhibition of renal interstitial fibrosis.

Using $\alpha$-SMA, CTGF, and E-cadherin as our indicators for renal cellular transdifferentiation and fibrosis and human renal proximal tubular epithelial HK-2 cells that are induced by parathyroid hormone (PTH) as our in vitro experimental model, we created the conditions mimicking RIF in a renal disease state in which expression of CTGF and $\alpha$-SMA is elevated through PTH stimulation. To date, there is no natural remedy that effectively blocks CTGF and $\alpha$-SMA in renal fibrosis. In this study, we investigated the ability of genistein to inhibit cell transdifferentiation through inhibition of CTGF and $\alpha$-SMA. Our finding provides the initial evidence and molecular mechanism by which genistein mediates inhibition of PTH-induced RIF.

\section{Material and methods}

\section{Reagents and equipment}

Genistein and PTH (1-84) were purchased from Sigma (USA). The CTGF polyclonal antibody was from R\&D systems (USA). The mouse $\alpha$-SMA monoclonal antibody was from Abcam. Fluorescein isothiocyanate (FITC) used as a secondary antibody was from Boster (China). F12/DMEM culture medium, Opti-MEM, reagents associated with cDNA synthesis and reverse transcription, TRIZOL, and Lipofectamine 2000 were from Invitrogen (USA). M-MLV reverse transcriptase, dNTP, random primers, RNase inhibitor, Pfu DNA polymerase, pGL3-basic vector, pGL3-CTGF promoter, pRL-SV40 plasmid, JM109 competent cells, and Dual-Luciferase Reporter Assay System were from Promega (USA). Lumat LB 9507 for detection of luciferase activity and the incubator for cultured cells (model 311) were manufactured by Thermo Forma (USA). The GDS 8000 image analysis system was manufactured by UVP (USA). The PCR thermal cycler was from Whatman Biometra (Germany).

\section{Cell culture and treatments}

Human renal proximal tubular epithelial HK-2 cells were obtained from the Institute of Basic Medical Sciences, Beijing, China. HK-2 cells were grown in DMEM/F-12 supplemented with $10 \%$ fetal bovine serum (FBS), penicillin and streptomycin in a humidified $5 \% \mathrm{CO}_{2}$ incubator at $37^{\circ} \mathrm{C}$. For treatment experiments, cells were plated on 6 -well plates following trypsin incubation and incubated for $24 \mathrm{~h}$ with $2 \mathrm{ml} /$ well DMEM/F12 medium containing no FBS. The relative molecular weight of genistein (Sigma) was 270.2 ( $\geq 98 \%$ purity). A total of $5 \mathrm{mg}$ of genistein was dissolved in $1.85 \mathrm{ml}$ of dimethyl sulfoxide (DMSO) to form a $10^{-2} \mathrm{~mol} / \mathrm{l}$ stock solution that was then diluted to $1-50 \mathrm{mmol} / \mathrm{l}$. To determine the effects of genistein on PTH-induced gene expressions of CTGF and $\alpha$-SMA, cells were treated with either DMSO, PTH (0.1 nM) alone, or various concentrations of genistein $(1,25,50,100 \mu \mathrm{M})$ along with $0.1 \mathrm{nM}$ PTH. Cells were pre-incubated with genistein for 30 min followed by PTH treatment for $48 \mathrm{~h}$.

\section{Detection of expression of CTGF mRNA levels by real-time RT-PCR}

Following treatments, cells were harvested and total RNA was immediately extracted using Trizol. Three $\mu \mathrm{g}$ of total RAN was used to synthesize firststrand DNA with reverse transcriptase according to the manufacturer's protocol (Invitrogen, USA). For CTGF gene expression analysis, the 211-bp DNA fragment was generated with the following primers: forward, 5'-GACCCAACTATGATTAGAGCCA-3', 
and reverse, 5'-CCGTCGGTACATACTCCACA-3'. The human $\beta$-actin RNA, used as an internal control gene, was amplified with sequence-specific primers (forward, 5' -CCTGTACGCCAACACAGTGC-3', and reverse, 5'-ATACTCCTGCTTGCTGATCC-3') to generate a 299-bp DNA fragment. Real-time PCR was conducted with a Green PCR Master Mix Kit (Shanghai Shine Co., China). Briefly, one microliter of firststrand CDNA and gene specific primers were used along with Hotstart Fluo-PCR Mix in a $20 \mu \mathrm{l} \mathrm{reac}-$ tion using a thermal profile as follows: $95^{\circ} \mathrm{C}$ for $5 \mathrm{~min}, 35$ cycles of $95^{\circ} \mathrm{C}$ for $10 \mathrm{~s}, 57^{\circ} \mathrm{C}$ for $15 \mathrm{~s}$ and $72^{\circ} \mathrm{C}$ for $20 \mathrm{~s}$ followed by a final extension for $10 \mathrm{~min}$ at $72^{\circ} \mathrm{C}$. Each sample was performed in triplicate and was quantified based on the formula $\Delta C t=C t_{(\mathrm{CTFG})}-C t_{(\beta \text {-actin) }}$. The PCR products $(4 \mu \mathrm{l})$ were further examined by agarose gel electrophoresis with $2 \%$ agarose gel to ensure that specific DNA fragments were generated.

\section{Western blot analysis}

Western blot analyses were performed for detection of $\alpha$-SMA, E-cadherin, and CTGF protein expression levels. Following HK-2 cell treatments, the cell lysates were prepared in 1 X RIPA lysis buffer, and protein concentrations for each sample were quantitated by Lowry assay with modification. Electrophoresis was conducted with $100 \mu \mathrm{g}$ cell lysate and the resolved protein was transferred onto a nitrocellulose membrane by using an electrotransfer apparatus. The membrane was blocked with $5 \%$ nonfat dry milk in TBST buffer at room temperature for $1 \mathrm{~h}$. The membrane was then incubated with the following primary antibodies: $\alpha$-SMA (1: 1000 dilution), E-cadherin (1: 2000 dilution), or CTGF (1: 1000 dilution) at $4^{\circ} \mathrm{C}$ overnight. The membrane was then washed for $30 \mathrm{~min}$ followed by incubation with horseradish peroxidaseconjugated secondary antibody (dilution $1: 3000$ ) for $30 \mathrm{~min}$ at $37^{\circ} \mathrm{C}$. The blot was visualized with diaminobenzidine (DAB)-hydrogen peroxide at room temperature following the washing step. The relative protein levels were determined by densitometry of the bands with SxImage software (Shanghai, China).

\section{Reporter assay for detection of the CTGF promoter activity}

The CTGF gene promoter was cloned using genomic DNA isolated from HK-2 cells with specific primers based on CTGF gene sequence (GenBank number AF316368) and designed by Primier 5 software. The CTGF promoter region was amplified by PCR and subcloned into a pGL3 basic vector following restriction enzyme digestions to obtain the pCTGF-Luc recombinant DNA. For transient transfections, HK-2 cells were transfected with either a pCTGF-luc plasmid or a pGL3 basic vector along with PRL-SV40 as an internal control for DNA transfection efficiency. The CTGF promoter activity was determined by the expression of firefly luciferase and was normalized to the Renilla luciferase levels by using a dual luciferase reporter assay kit (Promega).

\section{Immunofluorescence staining}

Immunofluorescence staining was used to determine the expression of $\alpha$-SMA. Cells were pre-treated with genistein $(100 \mu \mathrm{M})$ for 30 min followed by addition of PTH (0.1 nM) for $48 \mathrm{~h}$, then incubated with primary antibody mouse anti-human $\alpha$-SMA (dilution $1: 200$ ) for $60 \mathrm{~min}$ at $37^{\circ} \mathrm{C}$, with secondary antibody goat anti-mouse IgG-FITC (dilution $1: 100$ ) for $1 \mathrm{~h}$ at $37^{\circ} \mathrm{C}$. The cell nuclei were stained by adding DAPI with 3 min incubation at room temperature. Protein expression levels were visualized under a fluorescence microscope and photographs were recorded.

\section{Statistical analysis}

Each data point was repeated three times, and data are expressed as the mean \pm SD, and $t$-test and variance analysis were conducted using the statistical software SPSS12.0. Each treatment group was compared with the control group with Dunnett's $t$-test, and $p$ value $<0.05$ was used to define statistical significance.

\section{Results}

\section{Genistein inhibited PTH-induced $\alpha$-SMA expression in HK-2 cells}

Results of immunofluorescence staining revealed that the expression of $\alpha$-SMA was low in the control (PBS treatment) group of HK-2 cells. When cells were treated with $0.1 \mathrm{nM}$ PTH for $48 \mathrm{~h}, \alpha$-SMA expression levels were significantly increased; however, pretreatment of genistein for 30 min followed by PTH stimulation efficiently inhibited PTH-induced $\alpha$-SMA expression as shown in Figure 1.

\section{The effects of genistein on protein expression levels of $\alpha$-SMA and E-cadherin in HK-2 cells}

Similar to results of immunofluorescence, $\alpha$-SMA expression levels in HK-2 cells were elevated following 48-hour PTH stimulation detected by Western blot analysis. It is well established that a reduction of E-cadherin, a marker of epithelial cells for maintaining the adhesion properties in renal tubular epithelial cells, has been recognized as a molecular marker associated with EMT [9]; thus E-cadherin expression was examined in HK-2 cells. Conversely, E-cadherin protein levels were substantially decreased after PTH treatment. 

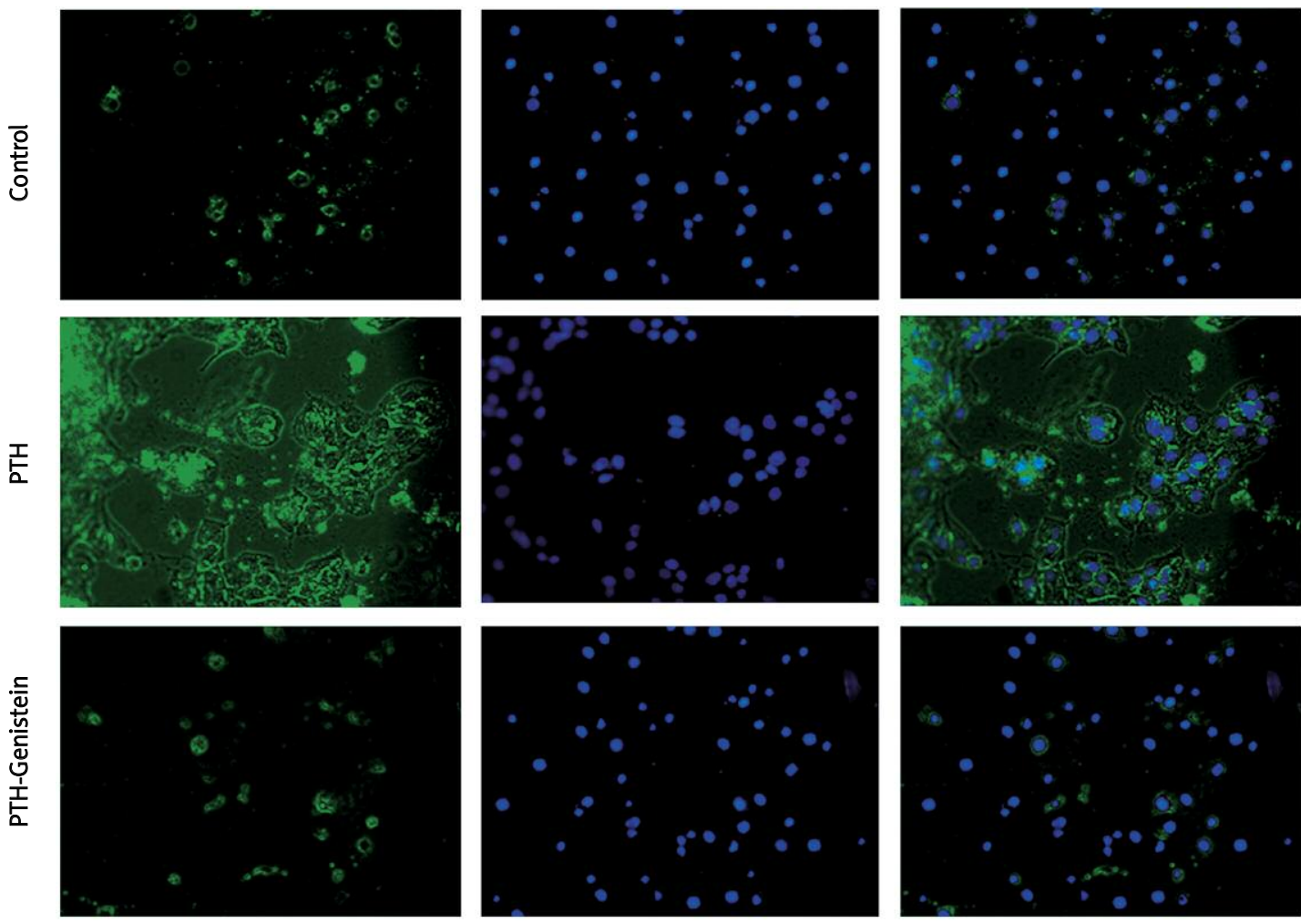

$\alpha-S M A$

DAPI

MERGE

Figure 1. The effect of genistein on PTH-induced $\alpha$-SMA expression in HK-2 cells

When cells were co-treated with both PTH and genistein, $\alpha$-SMA protein expression levels decreased and E-cadherin levels restored as a function of genistein concentration (Figure 2). The statistical significances are shown between PTH alone and co-treatment of PTH and genistein at genistein concentrations of 25,50 , and $100 \mu \mathrm{M}$ $(p<0.01)$.

The effects of genistein on PTH-induced RNA and protein expression profiles of CTGF

In normal conditions (the control group), there were basal CTGF mRNA expression levels. In comparison with the control group, PTH treatment $(0.1 \mathrm{nM})$ significantly induced CTGF mRNA detected by real time RT-PCR expression (3.95 \pm 0.18 vs. $0.54 \pm 0.07, p<0.01)$. When PTH was co-treated with genistein in HK-2 cells, genistein, with concentrations ranging from $25 \mu$ to $100 \mu \mathrm{M}$, effectively blocked PTH-induced CTGF expression $(p<0.01)$, as shown in Figure 3 A. Similarly, $0.1 \mathrm{nM}$ PTH substantially induced CTGF protein expression levels $(p<0.01)$. Low concentration of genistein $(5 \mu \mathrm{M})$ did not affect CTGF expression; however, a statistically significant decrease in CTGF protein levels was observed in the genistein pre-treatment group with genistein concentrations of 25,50 , and $100 \mu \mathrm{M}$ compared to the PTH-treated group $(p<0.01)$ (Figure 3 B).
The effects of genistein on PTH-induced CTGF promoter activity

Transient transfection experiments were performed using a plasmid containing the CTGF promoter connected with a luciferase basic vector (pCTGF-luc). We observed that firefly luciferase activities were dramatically elevated compared with that of a plain luciferase basic vector (pGL3-basic

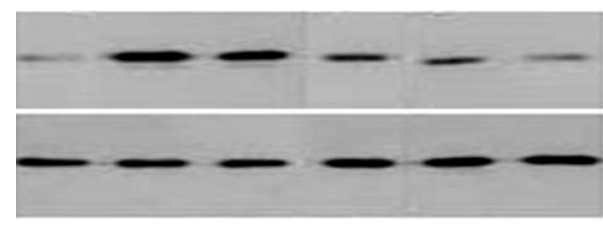
$\alpha-S M A$

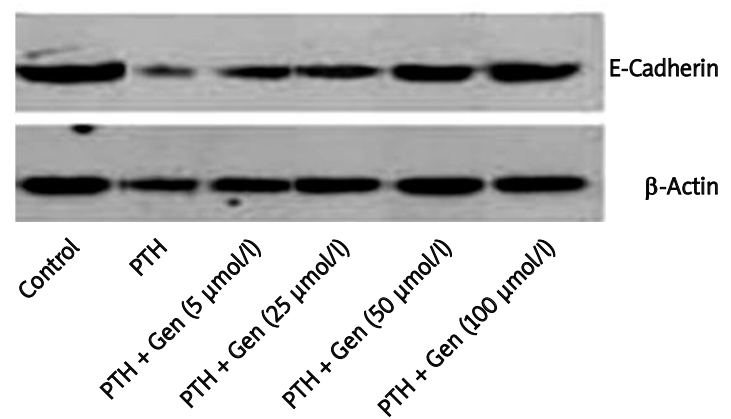

Figure 2. Expression of $\alpha-S M A$ and $E$-cadherin in response to genistein in HK-2 cells 

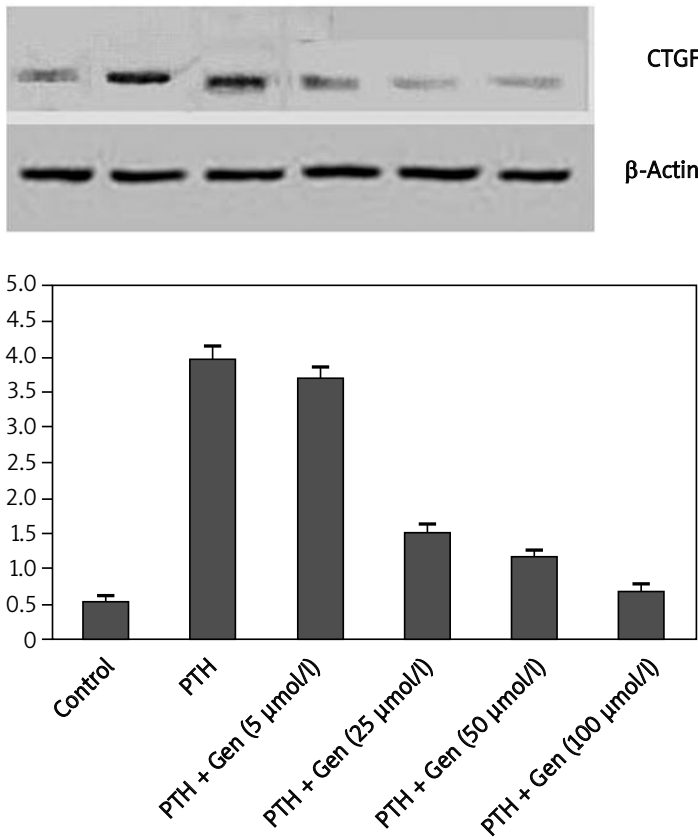

Figure 3. The effect of genistein on PTH-induced CTGF expression in HK-2 cells

vector) ( $61048.3 \pm 972.8$ vs. $58.3 \pm 6.0, p=0.0004)$ indicating that the CTGF promoter possesses functional activity for transcriptional activation. When transfected HK-2 cells were treated with PTH, luciferase activities were significantly increased compared with that of the control group (without PTH treatment) (1.888 \pm 0.078 vs. $0.989 \pm 0.030$, $p<0.01$ ), suggesting that the CTGF promoter activity can be induced by PTH. Furthermore, treatment of $100 \mu \mathrm{M}$ genistein can effectively block PTH-mediated CTGF promoter induction to a level close to the basal expression without PTH treatment (0.932 $\pm 0.032, p<0.01)$.

\section{Discussion}

Renal interstitial fibrosis (RIF) and epithelial transdifferentiation are recognized as key steps leading to end-stage renal disease. Parathyroid hormone (PTH) is a well-characterized endocrine regulator of mineral homeostasis and a common uremic toxin. The previous study in our group demonstrated that PTH can directly up-regulate expression of TGF- $\beta$ and fibronectin in rat glomerular mesangial cells [11]. We also established a strong line of evidence suggesting that through PTHrP [12], PTH triggered elevated expression of CTGF in renal proximal tubular cells [13]; these results indicate that PTH may play a pivotal role in the fibrotic process during chronic kidney disease. An increase in serum PTH in chronic kidney disease, known as secondary hyperparathyroidism, is an inevitable complication accompanying end-stage renal disease due to a prolonged decrease in serum $\mathrm{Ca}^{2+}$ and $1,25(\mathrm{OH})_{2} \mathrm{D}_{3}$ in chronic renal failure [14]. This secondary hyperparathyroidism involves increases in PTH gene expression, synthesis, and secretion, as well as proliferation of parathyroid cells leading to parathyroid hypertrophy [15]. Based on our previous study, PTH secretion also correlates with the expression of CTGF and $\alpha$-SMA [16]. Our current study focused on the tubular epithelial cells, especially in relation to the inflammatory and fibrogenic signaling that is activated after PTH insult to HK-2 cells, and more importantly, the ability of genistein to counteract PTH-induced effects. Our finding directly implicates a novel interaction between genistein and activated tubular epithelial HK-2 cells, suggesting that genistein can be used as a novel therapeutic agent against renal transdifferentiation and fibrogenesis.

In the past, much attention has been paid to searching for an effective means to control and reverse the disease stage of renal tubular epithelial cells. As a pro-fibrotic growth factor, TGF- $\beta$, a key cytokine involved in renal fibrogenesis, regulates extracellular matrix (ECM) and downstream target genes including CTGF [17]. Due to its anti-inflammatory properties, therapeutic strategies inhibiting TGF- $\beta$ actions have not achieved the expected beneficial effects [18, 19]. In the current study, we have used genistein to target the expression of CTGF, a novel fibrogenic regulator induced in various human kidney diseases and contributing to renal fibrosis and tubuloepithelial transdifferentiation [20]. Previous studies using antisense CTGF oligonucleotides have demonstrated inhibition of angiogenesis II-induced fibrosis as indicated by a decrease in fibronectin production [18] Combining our data with the fact that genistein blocks PTH-induced CTGF expression effectively in HK-2 cells, we predict that genistein may be a potent natural agent to inhibit CTGF inhibition and thus renal fibrosis, in vivo. The clinical study of genistein with patients who suffer chronic kidney disease will be needed to prove its efficacy. Moreover, the bioavailability of genistein as well as its pharmacokinetic profile in human metabolism requires further assessment. These data will give us a better understanding regarding the physiological concentration of genistein to reach its effectiveness in the target renal tissues.

As the most abundant isoflavone present in soybean and soy-based products, genistein has been reported to inhibit the growth of a wide range of cultured cancer cells, such as breast cancer, lymphoma, melanoma, and prostate cancer [7, 21]. The anti-tumorigenesis property of genistein might be associated with its ability to be a potent inhibitor of protein tyrosine kinase [22], DNA topoisomerase II [23], and ribosomal S6 kinase [24] although direct mechanisms are still unclear. Genistein possesses a weak estrogenic effect and non-specific tyrosine kinase inhibitory activity at pharmacological doses 
[25]. The role of isoflavones from soy proteins in the attenuating effect on glomerular injury has been proved previously [26]. A recent study by our group also demonstrated the ability of genistein to reduce renal tubular expansion and improve renal function in diabetic rats [27]. Because of its multitargeting properties, low toxicity and immediate availability, genistein has gained considerable attention for prevention and treatment of inflammationrelated symptoms. However, there is no report directly addressing whether genistein can exert its effect on inhibition of renal interstitial fibrosis. We provide strong evidence here that links genistein potential to block chronic renal disease: genistein effectively regulates $\alpha$-SMA and E-cadherin, molecules closely associated with EMA and RIF during renal disease, as well as CTGF, a key regulator of renal inflammatory and fibrogenic responses. This finding elucidates the key target molecules that genistein exerts its action on, but the detailed mechanism remains to be studied.

In conclusion, we conclude on a preliminary basis that genistein could inhibit expression of connective tissue growth factor as well as ameliorate epithelial-to-mesenchymal transition, which may be a novel therapeutic agent against renal transdifferentiation and fibrogenesis in PTH-induced endstage renal disease.

\section{Acknowledgments}

This study was supported by: Chinese Post-Doctoral Scientific Foundation, No. 2012M521928 and Shandong Provicial oustanding young scientist fund, No. BS2012Y041.

\section{References}

1. Liu Y. Epithelial to mesenchymal transition in renal fibrogenesis: pathologic significance, molecular mechanism, and therapeutic intervention. J Am Soc Nephrol 2004; 15: 1-12.

2. Abbate M, Zoja C, Rottoli D, Corna D, Tomasoni S, Remuzzi G. Proximal tubular cells promote fibrogenesis by TGF-beta1-mediated induction of peritubular myofibroblasts. Kidney Int 2002; 61: 2066-77.

3. Border WA, Noble NA. TGF-beta in kidney fibrosis: a target for gene therapy. Kidney Int 1997; 51: 1388-96.

4. Ito Y, Aten J, Bende RJ, et al. Expression of connective tissue growth factor in human renal fibrosis. Kidney Int 1998; 53: 853-61.

5. Strutz F, Okada H, Lo CW, et al. Identification and characterization of a fibroblast marker FSP1. J Cell Biol 1995; 130: 393-405.

6. Iwano M, Plieth D, Danoff TM, et al. Evidence that fibroblasts derive from epithelium during tissue fibrosis. J Clin Invest 2002; 110: 341-50.

7. Gupta SC, Kim JH, Kannappan R, Reuter S, Dougherty PM, Aggarwal BB. Role of nuclear factor kappaB-mediated inflammatory pathways in cancer-related symptoms and their regulation by nutritional agents. Exp Biol Med 2011; 236: 658-71.
8. Si H, Liu D. Phytochemical genistein in the regulation of vascular function: new insights. Curr Med Chem 2007; 14: 2581-9.

9. Verdrengh M, Jonsson IM, Holmdahl R, Tarkowski A. Genistein as an anti-inflammatory agent. Inflamm Res 2003; 52: 341-6.

10. Cano A, Pérez-Moreno MA, Rodrigo I, et al. The transcription factor snail controls epithelial-mesenchymal transitions by repressing E-cadherin expression. Nat Cell Biol 2000; 2: 76-83.

11. Zhan F, Yuan W. The effects of parathyroid hormone on synthesis and secretion of fibrogenic proteins in mesangial cells. Chin J Nephrol 2002; 18: 442-3.

12. Largo R, Gomez-Garre D, Santos $S$, et al. Renal expression of parathyroid hormone-related protein (PTHrP) and PTH/PTHrP receptor in a rat model of tubulointerstitial damage. Kidney Int 1999; 55: 82-90.

13. Guo Y, Yuan W, Wang L, Shang M, Peng Y. Parathyroid hormone-potentiated connective tissue growth factor expression in human renal proximal tubular cells through activating the MAPK and NF-kappaB signalling pathways. Nephrol Dial Transplant 2011; 26: 839-47.

14. Portale AA, Morris RC Jr. Pathogenesis of secondary hyperparathyroidism in chronic renal insufficiency. Miner Electrolyte Metab 1991; 17: 211-20.

15. Silver J, Kilav R, Naveh-Many T. Mechanisms of secondary hyperparathyroidism. Am J Physiol Renal Physiol 2002; 283: F367-76.

16. Guo Y, Yuan W. The effects of parathyroid hormone on transdifferentiation and expression of connective tissue growth factor in human proximal tubular epithelium cells. Chin J Nephrol 2008; 24: 332-6.

17. Ruiz-Ortega M, Ruperez M, Esteban V, et al. Angiotensin II: a key factor in the inflammatory and fibrotic response in kidney diseases. Nephrol Dial Transplant 2006; 21: 16-20.

18. Ruperez M, Lorenzo O, Blanco-Colio LM, et al. The connective tissue growth factor is a mediator of angiotensin II-induced fibrosis. Circulation 2003; 108: 1499-509.

19. Grainger DJ. Transforming growth factor beta and atherosclerosis: so far, so good for the protective cytokine hypothesis. Arterioscler Thromb Vasc Biol 2004; 24: 399-404.

20. Perbal B. CCN proteins: multifunctional signalling regulators. Lancet 2004; 363: 62-4.

21. Piotrowska E, Jakobkiewicz-Banecka J, Maryniak A, et al. Two-year follow-up of Sanfilippo sisease patients treated with a genistein-rich isoflavone extract: assessment of effects on cognitive functions and general status of patients. Med Sci Monit 2011; 17: CR196-202.

22. Akiyama T, Ishida J, Nakagawa S, et al. Genistein, a specific inhibitor of tyrosine-specific protein kinase. J Biol Chem 1987; 262: 5592-5.

23. Schmidt F, Knobbe CB, Frank B, Wolburg H, Weller M. The topoisomerase II inhibitor, genistein, induces $\mathrm{G} 2 / \mathrm{M}$ arrest and apoptosis in human malignant glioma cell lines. Oncol Rep 2008; 19: 1061-6.

24. Linassier C, Pierre M, Le Peco JB, Pierre J. Mechanisms of action in NIH-3T3 cells of genistein, an inhibitor of EGF receptor tyrosine kinase activity. Biochem Pharmacol 1990; 39: 187-93.

25. Valsecchi AE, Franchi S, Panerai AE, Sacerdote P, Trovato AE, Colleoni M. Genistein, a natural phytoestrogen from soy, relieves neuropathic pain following chronic constriction sciatic nerve injury in mice: anti-inflammatory and antioxidant activity. J Neurochem 2008; 107: 230-40. 
26. Sakemi T, Ikeda Y, Shimazu K, Uesugi T. Attenuating effect of a semipurified alcohol extract of soy protein on glomerular injury in spontaneous hypercholesterolemic male Imai rats. Am J Kidney Dis 2001; 37: 832-7.

27. Yuan WJ, Jia FY, Meng JZ. Effects of genistein on secretion of extracellular matrix components and transforming growth factor beta in high-glucose-cultured rat mesangial cells. J Artif Organs 2009; 12: 242-6. 